\title{
A Perceptually Relevant No-Reference Blockiness Metric Based on Local Image Characteristics
}

\author{
Hantao Liu (EURASIP Member) ${ }^{1}$ and Ingrid Heynderickx ${ }^{1,2}$ \\ ${ }^{1}$ Department of Mediamatics, Delft University of Technology, 2628 CD Delft, The Netherlands \\ ${ }^{2}$ Group Visual Experiences, Philips Research Laboratories, 5656 AA Eindhoven, The Netherlands
}

Correspondence should be addressed to Hantao Liu, hantao.liu@tudelft.nl

Received 4 July 2008; Revised 20 December 2008; Accepted 21 January 2009

Recommended by Dimitrios Tzovaras

A novel no-reference blockiness metric that provides a quantitative measure of blocking annoyance in block-based DCT coding is presented. The metric incorporates properties of the human visual system (HVS) to improve its reliability, while the additional cost introduced by the HVS is minimized to ensure its use for real-time processing. This is mainly achieved by calculating the local pixel-based distortion of the artifact itself, combined with its local visibility by means of a simplified model of visual masking. The overall computation efficiency and metric accuracy is further improved by including a grid detector to identify the exact location of blocking artifacts in a given image. The metric calculated only at the detected blocking artifacts is averaged over all blocking artifacts in the image to yield an overall blockiness score. The performance of this metric is compared to existing alternatives in literature and shows to be highly consistent with subjective data at a reduced computational load. As such, the proposed blockiness metric is promising in terms of both computational efficiency and practical reliability for real-life applications.

Copyright (c) 2009 H. Liu and I. Heynderickx. This is an open access article distributed under the Creative Commons Attribution License, which permits unrestricted use, distribution, and reproduction in any medium, provided the original work is properly cited.

\section{Introduction}

Objective metrics, which serve as computational alternatives for expensive image quality assessment by human subjects, aimed at predicting perceived image quality aspects automatically and quantitatively. They are of fundamental importance to a broad range of image and video processing applications, such as for the optimization of video coding or for real-time quality monitoring and control in displays $[1,2]$. For example, in the video chain of current TVsets, various objective metrics, which determine the quality of the incoming signal in terms of blockiness, ringing, blur, and so forth and adapt the parameters in the video enhancement algorithms accordingly, are implemented to enable an improved overall perceived quality for the viewer.

In the last decades, a considerable amount of research has been carried out on developing objective image quality metrics, which can be generally classified into two categories: full-reference (FR) metrics and no-reference (NR) metrics [1]. The FR metrics are based on measuring the similarity or fidelity between the distorted image and its original version, which is considered as a distortion-free reference. However, in real-world applications the reference is not always fully available; for example, the receiving end of a digital video chain usually has no access to the original image. Hence, objective metrics used in these types of applications are constrained to a no-reference approach, which means that the quality assessment relies on the reconstructed image only. Although human observers can easily judge image quality without any reference, designing NR metrics is still an academic challenge mainly due to the limited understanding of the human visual system [1]. Nevertheless, since the structure information of various image distortions is well known, NR metrics designed for specific quality aspects rather than for overall image quality are simpler, and therefore, more realistic [2].

Since the human visual system (HVS) is the ultimate assessor of most visual information, taking into account the way human beings perceive quality aspects, while removing perceptual redundancies, can be greatly beneficial for matching objective quality prediction to human, perceived quality [3]. This statement is adequately supported by the observed 
shortcoming of the purely pixel-based metrics, such as the mean square error (MSE) and peak signal-to-noise ratio (PSNR). They insufficiently reflect distortion annoyance to the human eye, and thus often exhibit a poor correlation with subjective test results (e.g., in [1]). The performance of these metrics has been enhanced by incorporating certain properties of the HVS (e.g., in [4-7]). But since the HVS is extremely complex, an objective metric based on a model of the HVS often is computationally very intensive. Hence, to ensure that an HVS-based objective metric is applicable to real-time processing, investigations should be carried out to reduce the complexity of the HVS model as well as of the metric itself without significantly compromising the overall performance.

One of the image quality distortions for which several objective metrics have been developed is blockiness. A blocking artifact manifests itself as an artificial discontinuity in the image content and is known to be the most annoying distortion at low bit-rate DCT coding [8]. Most objective quality metrics either require a reference image or video (e.g., in [5-7]), which restricts their use in real-life applications, or lack an explicit human vision model (e.g., in $[9,10]$ ), which limits their reliability. Apart from these metrics, noreference, blockiness metrics, including certain properties of the HVS are developed. Recently, a promising approach, which we refer to as feature extraction method, is proposed in $[11,12]$, where the basic idea is to extract certain image features related to the blocking artifact and to combine them in a quality prediction model with the parameters estimated from subjective test data. The stability of this method, however, is uncertain since the model is trained with a limited set of images only, and its reliability to other images is not proved yet.

A no-reference blockiness metric can be formulated either in the spatial domain or in the transform domain. The metrics described, for example, in $[13,14]$ are implemented in the transform domain. In [13], a 1-D absolute difference signal is combined with luminance and texture masking, and from that blockiness is estimated as the peaks in the power spectrum using FFT. In this case, the FFT has to be calculated many times for each image, which is therefore very expensive. The algorithm in [14] computes the blockiness as a result of a 2-D step function weighted with a measure of local spatial masking. This metric requires the access to the DCT encoding parameters, which are, however, not always available in practical applications.

In this paper, we rely on the spatial domain approach. The generalized block-edge impairment metric (GBIM) [15] is the most well-known metric in this domain. GBIM expresses blockiness as the interpixel difference across block boundaries scaled with a weighting function, which simply measures the perceptual significance of the difference due to local spatial masking of the HVS. The total amount of blockiness is then normalized by the same measure calculated for all other pixels in an image. The main drawbacks for GBIM are (1) the interpixel difference characterizes the block discontinuity not to the extent that local blockiness is sufficiently reliably predicated; (2) the HVS model includes both luminance masking and texture masking in a single weighting function, and efficient integration of different masking effects is not considered, hence, applying this model in a blockiness metric may fail in assessing demanding images; (3) the metric is designed such that the human vision model needs to be calculated for every pixel in an image, which is computationally very expensive. A second metric using the spatial domain is based on a locally adaptive algorithm [16] and is hereafter referred to as LABM. It calculates a blockiness metric for each individual coding block in an image and simultaneously estimates whether the blockiness is strong enough to be visible to the human eye by means of a just-noticeable-distortion (JND) profile. Subsequently, the local metric is averaged over all visible blocks to yield a blockiness score. This metric is promising and potentially more accurate than GBIM. However, it exhibits several drawbacks: (1) the severity of blockiness for individual artifacts might be under- or overestimated by providing an averaged blockiness value for all artifacts within this block; (2) calculating an accurate JND profile which provides a visibility threshold of a distortion due to masking is complex, and it cannot predict perceived annoyance above threshold; (3) the metric needs to estimate the JND for every pixel in an image, which largely increases the computational cost.

Calculating the blockiness metric only at the expected block edges, and not at all pixels in an image, strongly reduces the computational power, especially when a complex HVS is involved. To ensure that the metric is calculated at the exact position of the block boundaries, a grid detector is needed since in practice deviations in the blocking grid might occur in the incoming signal, for example, as a consequence of spatial scaling $[9,17,18]$. Without this detection phase, noreference metrics might turn out to be useless, as blockiness is calculated at wrong pixel positions.

In this paper, a novel algorithm is proposed to quantify blocking annoyance based on its local image characteristics. It combines existing ideas in literature with some new contributions: (1) a refined pixel-based distortion measure for each individual blocking artifact in relation to its direct vicinity; (2) a simplified and more efficient visual masking model to address the local visibility of blocking artifacts to the human eye; (3) the calculation of the local pixelbased distortion and its visibility on the most relevant stimuli only, which significantly reduces the computational cost. The resulting metric yields a strong correlation with subjective data. The rest of the paper is organized as follows. Section 2 details the proposed algorithm, Section 3 provides and discusses the experimental results, and the conclusions are drawn in Section 4.

\section{Description of the Algorithm}

The schematic overview of the proposed approach is illustrated in Figure 1 (the first outline of the algorithm was already described in [19]). Initially, a grid detector is adopted in order to identify the exact position of the blocking artifacts. After locating the artifacts, local processing is carried out to individually examine each detected blocking artifact by analyzing its surrounding content to a limited 


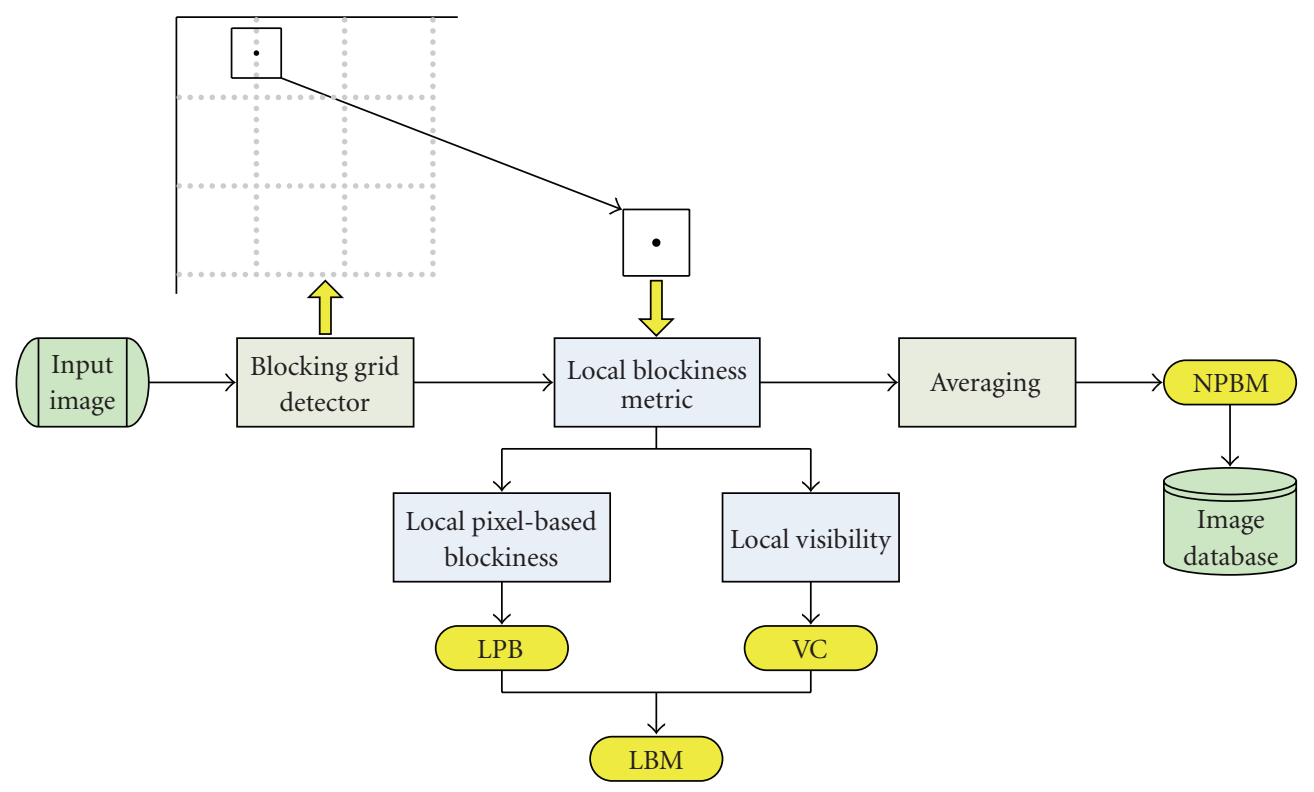

FIgURE 1: Schematic overview of the proposed approach.

extent. This local calculation consists of two parallel steps: (1) measuring the degree of local pixel-based blockiness (LPB); (2) estimating the local visibility of the artifact to the human eye and outputting a visibility coefficient (VC). The resulting LPB and VC are integrated into a local blockiness metric (LBM). Finally, the LBM is averaged over the blocking grid of the image to produce an overall score of blockiness assessment (i.e., NPBM). The whole process is calculated on the luminance channel only in order to further reduce the computational load. The algorithm is performed for the blockiness once in horizontal direction (i.e., $\mathrm{NPBM}_{h}$ ) and once in vertical direction $\mathrm{NPBM}_{v}$. From both values, the average is calculated assuming that the human sensitivity to horizontal and vertical blocking artifacts is equal.

2.1. Blocking Grid Detection. Since the arbitrary grid problem has emerged as a crucial issue especially for no-reference blockiness metrics, where no prior knowledge on grid variation is available, a grid detector is required in order to ensure a reliable metric $[9,18]$. Most, if not all, of the existing blockiness metrics make the strong assumption that the grid exists of blocks: $8 \times 8$ pixels, starting exactly at the top-left corner of an image. However, this is not necessarily the case in real-life applications. Every part of a video chain, from acquisition to display, may induce deviations in the signal, and the decoded images are often scaled before being displayed. As a result, grids are shifted, and the block size is changed.

Methods, as, for example, in [13, 17] employ a frequencybased analysis of the image to detect the location of blocking artifacts. These approaches, due to the additional signal transform involved, are often computationally inefficient. Alternatives in the spatial domain can be found in $[9,18]$. They both map an image into a one-dimensional signal profile. In [18], the block size is estimated using a rather complex maximum-likelihood method, and the grid offset is not considered. In [9], the block size and the grid offset are directly extracted from the peaks in the 1-D signal by calculating the normalized gradient for every pixel in an image. However, spurious peaks in the 1-D signal as a result of edges from objects may occur and consequently yield possible detection errors. In this paper, we further rely on the basic ideas of both $[9,18]$, but implement them by means of a simplified calculation of the 1-D signal and by extracting the block size and the grid offset using DFT of the 1-D signal. The entire procedure is performed once in horizontal and once in vertical directions to address a possible asymmetry in the blocking grid.

2.1.1. 1-D Signal Extraction. Since blocking artifacts regularly manifest themselves as spatial discontinuities in an image, their behavior can be effectively revealed through a 1-D signal profile, which is simply formed calculating the gradient along one direction (e.g., horizontal direction) and then summing up the results along the other direction (e.g., vertical direction). We denote the luminance channel of an image signal of $M \times N$ (height $\times$ width) pixels as $I(i, j)$ for $i \in[1, M], j \in[1, N]$, and calculate the gradient map $G_{h}$ along the horizontal direction

$$
G_{h}(i, j)=|I(i, j+1)-I(i, j)|, \quad j \in[1, N-1] .
$$

The resultant gradient map is reduced to a 1-D signal profile $S_{h}$ by summing $G_{h}$ along the vertical direction

$$
S_{h}(j)=\sum_{i=1}^{M} G_{h}(i, j) .
$$

2.1.2. Block Size Extraction. Based on the fact that the amount of energy present in the gradient at the borders 
of coding blocks is greater than that in the intermediate positions blocking artifacts, if existing, are present as a periodic impulse train of signal peaks. These signal peaks can be further enhanced using some form of spatial filtering, which makes the peaks stand out from their vicinity. In this paper, a median filter is used. Then a promoted 1-D signal profile $P S_{h}$ is obtained simply subtracting from $S_{h}$ its median-filtered version $M S_{h}$ :

$$
\begin{gathered}
P S_{h}(j)=S_{h}(j)-M S_{h}(j), \\
M S_{h}(j)=\operatorname{Median}\left\{S_{h}(j-k), \ldots, S_{h}(j), \ldots, S_{h}(j+k)\right\},
\end{gathered}
$$

where the size of the median filter $(2 k+1)$ depends on $N$. In our experiments, $N$ is, for example, 384, and then $k$ is 4. The resulting 1-D signal profile $P S_{h}$ intrinsically reveals the blocking grid as an impulse train with a periodicity determined by the block size. However, in demanding conditions, such as for images with many object edges, the periodicity in the regular impulses might be masked by noise as a result of image content. This potentially makes locating the required peaks and estimating their periodicity more difficult. The periodicity of the impulse train, corresponding to the block size, is more easily extracted from the 1-D signal $P S_{h}$ in the frequency domain using the discrete Fourier transform (DFT).

2.1.3. Grid Offset Extraction. After the block size (i.e., $p$ ) is determined, the offset of the blocking grid can be directly retrieved from the signal $P S_{h}$, in which the peaks are located at multiples of the block size. Thus, a simple approach based on calculating the accumulative value of grid peaks with a possible offset $\Delta x$ (e.g., $\Delta x=0:(p-1)$ with the periodic feature in mind) is proposed. For each possible offset value $\Delta x$, the accumulator is defined as

$$
A(\Delta x)=\sum_{i=1}^{[N / p]-1} P S_{h}(\Delta x+p \cdot i), \quad \Delta x \in[0, p-1] .
$$

The offset is determined as

$$
A(\Delta x)=\operatorname{MAX}[A(0) \cdots A(p-1)] .
$$

Based on the results of the block size and grid offset, the exact position of blocking artifacts can be explicitly extracted.

2.1.4. An Example. A simple example is given in Figure 2, where the input image "bikes" of $128 \times 192$ pixels is JPEGcompressed using a standard block size of $8 \times 8$ pixels. The displayed image is synthetically upscaled with a scaling factor $2 \times 2$ and shifted by 8 pixels both from left to right and from top to bottom. As a result, the displayed image size is $256 \times 384$ pixels, the block size $16 \times 16$ pixels, and the grid starts at pixel position $(8,8)$ instead of at the origin $(0,0)$, as shown in Figure 2(a). The proposed algorithm toward a 1-D signal profile is illustrated in Figure 2(b). Figure 2(c) shows the magnitude profile of the DFT applied to the signal PS. It allows extraction of the period $p$ (i.e., $p=1 / 0.0625=16$ pixels), which is maintained over the whole frequency range. Based on the detected block size $p=16$, the grid offset is calculated as $\Delta x=8$. Then the blocking grid can be determined, as shown in Figure 2(d).

2.2. Local Pixel-Based Blockiness Measure. Since blocking artifacts intrinsically are a local phenomenon, their behavior can be reasonably described at a local level, indicating the visual strength of a distortion within a local area of image content. Based on the physical structure of blocking artifacts as a spatial discontinuity, this can be simply accomplished relating the energy present in the gradient at the artifact with the energy present in the gradient within its vicinity. This local distortion measure (LDM) purely based on pixel information can be formulated as

$$
\operatorname{LDM}(k)=\frac{E^{k}(i, j)}{f\left[E^{V(k)}(i, j)\right]}, \quad k=1, \ldots, n,
$$

where $f[\cdot]$ indicates the pooling function, for example, $\Sigma$, mean, or L2-norm, $E^{k}$ indicates the gradient energy calculated for each individual artifact, $E^{V(k)}$ indicates the gradient energy calculated at the pixels in the direct vicinity of this artifact, and $n$ is the total number of blocking artifacts in an image. Since the visual strength of a block discontinuity is primarily affected by its local surroundings of limited extent, this approach is potentially more accurate than a global measure of blockiness (e.g., $[9,15])$, where the overall blockiness is assessed by the ratio of the averaged discontinuities on the blocking grid and the averaged discontinuities in pixels which are not on the blocking grid. Furthermore, the local visibility of a distortion due to masking can now be easily incorporated, with the result that it is only calculated at the location of the blocking artifacts. This means that modeling the HVS on nonrelevant pixels is eliminated as compared to the global approach (e.g., [15]).

In this paper, we rely on the interblock difference defined in [16] and extend the idea by reducing the dimension of the blockiness measure from a signal block to an individual blocking artifact. As such, the local distortion measure (LDM) is implemented on the gradient map, resulting in local pixel-based blockiness (LPB). The LPB quantifies the blocking artifact at pixel location $(i, j)$ as

$$
\mathrm{LPB}_{h}(i, j)= \begin{cases}\omega \times \mathrm{BG}_{h} & \text { if } \mathrm{NBG}_{h}=0, \mathrm{BG}_{h} \neq 0, \\ \frac{\mathrm{BG}_{h}}{\mathrm{NBG}_{h}} & \text { if } \mathrm{NBG}_{h} \neq 0, \\ 0 & \text { if } \mathrm{NBG}_{h}=0, B G_{h}=0,\end{cases}
$$

where $\mathrm{BG}_{h}$ and $\mathrm{NBG}_{h}$ are

$$
\begin{gathered}
\mathrm{BG}_{h}=G_{h}(i, j), \\
\mathrm{NBG}_{h}=\frac{1}{2 n} \sum_{x=-n, \ldots, n, x \neq 0} G_{h}(i, j+x) .
\end{gathered}
$$

The definition of the LPB is further explained as follows:

(1) The template addressing the direct vicinity is defined as a 1-D element including $\mathrm{n}$ adjacent pixels to the 


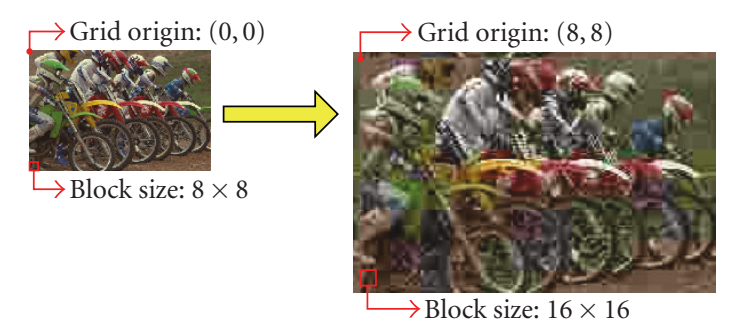

(a) Input image (left) and displayed image (right)

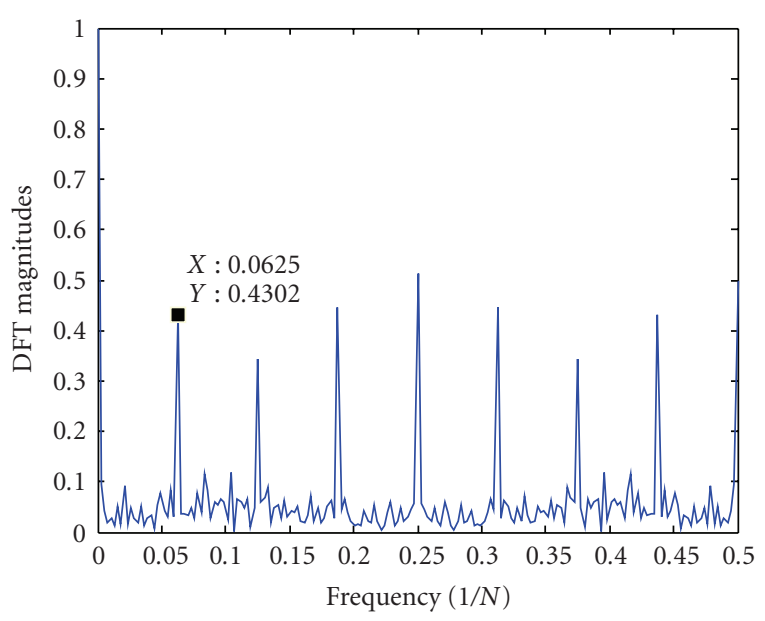

(c) DFT magnitudes of PS in (b)

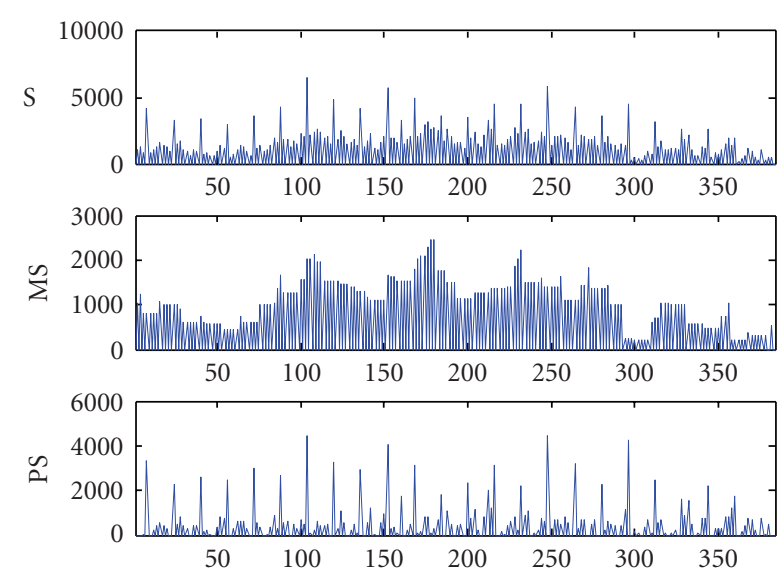

(b) 1-D signal formation: S, MS and PS are calculated according to (2) and (3) for the displayed image in (a) along the horizontal direction

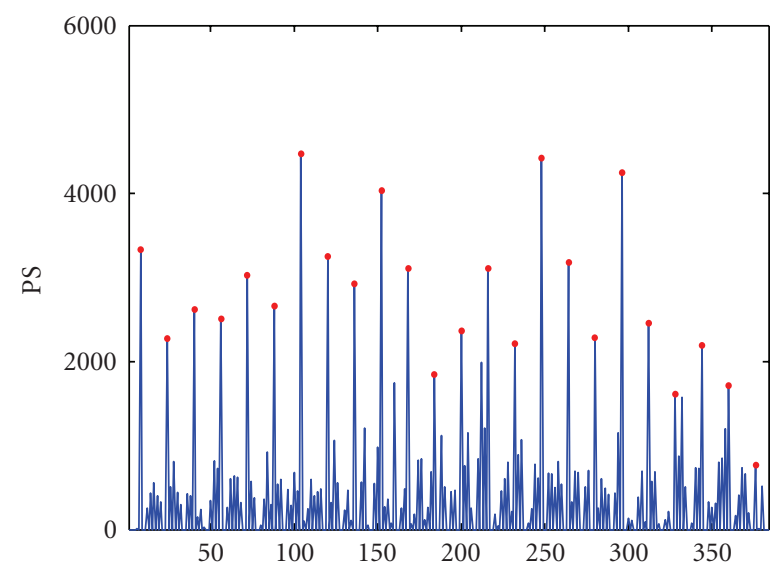

(d) Blocking grid detected from the displayed image in (a) along the horizontal direction

FIGURE 2: Blocking grid detection: an example.

left and to the right of an artifact. The size of the template $(2 n+1)$ is designed to be proportional to the detected block size $\mathrm{p}$ (e.g., $n=p / 2$ ), taking into account possible scaling of the decoded images. An example of the template is shown in Figure 3, where two adjacent $8 \times 8$ blocks (i.e., $\mathrm{A}$ and $\mathrm{B}$ ) are extracted from a real JPEG image.

(2) $\mathrm{BG}_{h}$ denotes the local energy present in the gradient at the blocking artifact, and $\mathrm{NBG}_{h}$ denotes the averaged gradient energy over its direct vicinity. If $\mathrm{NBG}_{h}=0$, only the value of $\mathrm{BG}_{h}$ determines the local pixel-based blockiness. In this case, $\mathrm{LPB}_{h}=0$ (i.e., $\mathrm{BG}_{h}=0$ ) means there is no block discontinuity appearing, and the blocking artifact is spurious. $\mathrm{LPB}_{h}=\omega \times \mathrm{BG}_{h}$ (i.e., $\mathrm{BG}_{h} \neq 0$ ) means the artifact exhibits a severe extent of blockiness, and $\omega(\omega=1$ in our experiments) is used to adjust the amount of gradient energy. If $\mathrm{NBG}_{h} \neq 0$, the local pixel-based blockiness is simply calculated as the ratio of $\mathrm{BG}_{h}$ over $\mathrm{NBG}_{h}$.

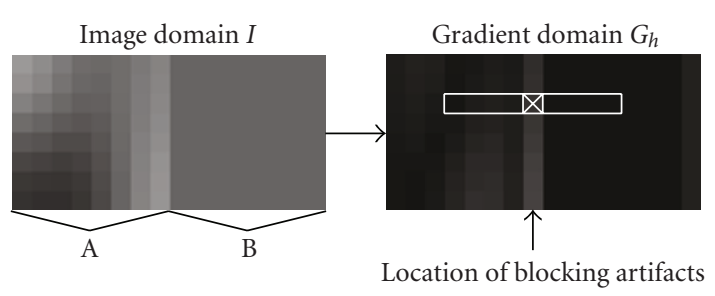

FIGURE 3: Local pixel-based blockiness (LPM).

(3) The local pixel-based blockiness $\mathrm{LPB}_{h}$ is specified in (7) to (8) for a block discontinuity along the horizontal direction. The measure of $\mathrm{LPB}_{v}$ for vertical blockiness can be easily defined in a similar way. The calculation is then performed within a vertical 1-D template.

2.3. Local Visibility Estimation. To predict perceived quality, objective metrics based on models of the human visual system are potentially more reliable $[3,20]$. However, from 
a practical point of view, it is highly desirable to reduce the complexity of the HVS model without compromising its abilities. In this paper, a simplified human vision model based on the spatial masking properties of the HVS is proposed. It adopts two fundamental characteristics of the HVS, which affect the visibility of an artifact in the spatial domain: (1) the averaged background luminance surrounding the artifact; (2) the spatial nonuniformity in the background luminance $[20,21]$. They are known as luminance masking and texture masking, respectively, and both are highly relevant to the perception of blocking artifacts.

Various models of visual masking to quantify the visibility of blocking artifacts in images have been proposed in literature $[7,11,15,21,22]$. Among these models, there are two widely used ones: the model used in GBIM [15] and the just-noticeable-distortion (JND) profile model used in [21]. Their disadvantages have already been pointed out in Section 1. Our proposed model is illustrated in Figure 4. Both texture and luminance masking are implemented by analyzing the local signal properties within a window, representing the local surrounding of a blocking artifact. A visibility coefficient as a consequence of masking (i.e., $\mathrm{VC}_{t}$ and $\mathrm{VC}_{l}$, resp.) is calculated using spatial filtering followed by a weighting function. Then, both coefficients are efficiently combined into a single visibility coefficient (VC), which reflects the perceptual significance of the artifact quantitatively.

2.3.1. Local Visibility Due to Texture Masking. Figure 5 shows an example of texture masking on blocking artifacts, where "a" and " $b$ " are patterns including 4 adjacent blocks of $8 \times 8$ pixels extracted from a JPEG-coded image. As can be seen from the right-hand side of Figure 5, pattern "a" and pattern "b" both intrinsically exhibit block discontinuities. However, as shown on the left-hand side of Figure 5, the block discontinuities in pattern "b" are perceptually masked by its nonuniform background, while the block discontinuities in pattern "a" are much more visible as it is in a flat background. Therefore, texture masking can be estimated from the local background activity [20]. In this paper, texture masking is modeled calculating a visibility coefficient $\left(\mathrm{VC}_{t}\right)$, indicating the degree of texture masking. The higher the value of this coefficient, the smaller the masking effect, and hence, the stronger the visibility of the artifact is. The procedure of modeling texture masking comprises three steps.

(i) Texture detection: calculate the local background activity (nonuniformity).

(ii) Thresholding: a classification scheme to capture the active background regions.

(iii) Visibility transform function (VTF): obtain a visibility coefficient $\left(\mathrm{VC}_{t}\right)$ based on the HVS characteristics for texture masking.

Texture detection can be performed convolving the signal with some form of high-pass filter. One of the Laws' texture energy filters [23] is employed here in a slightly modified form. As shown in Figure 6, T1 and T2 are used to measure the background activity in horizontal and vertical directions, respectively. A predefined threshold $\mathrm{Thr}$ ( $\mathrm{Thr}=0.15$ in our experiments) is applied to classify the background into "flat" or "texture," resulting in an activity value $I_{t}(i, j)$, which is given by

$$
\begin{aligned}
I_{t}(i, j) & = \begin{cases}0 & \text { if } t(i, j)<\mathrm{Thr}, \\
t(i, j) & \text { otherwise, }\end{cases} \\
t(i, j) & =\frac{1}{48} \sum_{x=1}^{5} \sum_{y=1}^{5} I(i-3+x, j-3+y) \cdot T(x, y),
\end{aligned}
$$

where $I(i, j)$ denotes the pixel intensity at location $(i, j), T$ is chosen as $T 1$ for texture calculation in horizontal direction, and $T 2$ in vertical direction. It should be noted that splitting up the calculation in horizontal and vertical directions, and using a modified version of the texture energy filter, in which some template coefficients are removed, can be done having the application of a blockiness metric in mind. The texture filters need to be adopted in case of extending these ideas to other objective metrics.

A visibility transform function (VTF) is proposed in accordance to human perceptual properties, which means that the visibility coefficient $\operatorname{VC}_{t}(i, j)$ is inversely proportional (nonlinear) to the activity value $I_{t}(i, j)$. Figure 6 shows an example of such a transform function, which can be defined as

$$
\mathrm{VC}_{t}(i, j)=\frac{1}{\left(1+I_{t}(i, j)\right)^{\alpha}},
$$

where $\operatorname{VC}_{t}(i, j)=1$, when the stimulus is in a "flat" background, and $\alpha>1(\alpha>5$ in our experiments $)$ is used to adjust the nonlinearity. This shape of the VTF is an approximation, considered to be good enough.

2.3.2. Local Visibility due to Luminance Masking. In many psychovisual experiments, it was found that the human visual system' sensitivity to variations in luminance depends on (is a nonlinear function of) the local mean luminance [7, 20, 21, 24]. Figure 7 shows an example of luminance masking on blocking artifacts, where "a" and "b" are synthetic patterns, each of which includes 2 adjacent blocks with different gray-scale levels. Although the intensity difference between the two blocks is the same in both patterns, the block discontinuity of pattern " $b$ " is much more visible than that in pattern "a" due to the difference in background luminance. In this paper, luminance masking is modeled based on two empirically driven properties of the HVS: (1) a distortion in a dark surrounding tends to be less visible than one in a bright surrounding $[7,21]$ and (2) a distortion is most visible for a surrounding with an averaged luminance value between 70 and 90 (centered approx. at 81) in 8 bits grayscale images [24]. The procedure of modeling luminance masking consists of two steps.

(i) Local luminance detection: calculate the localaveraged background luminance.

(ii) Visibility transform function (VTF): obtain a visibility coefficient $\left(\mathrm{VC}_{l}\right)$ based on the HVS characteristics for luminance masking. 


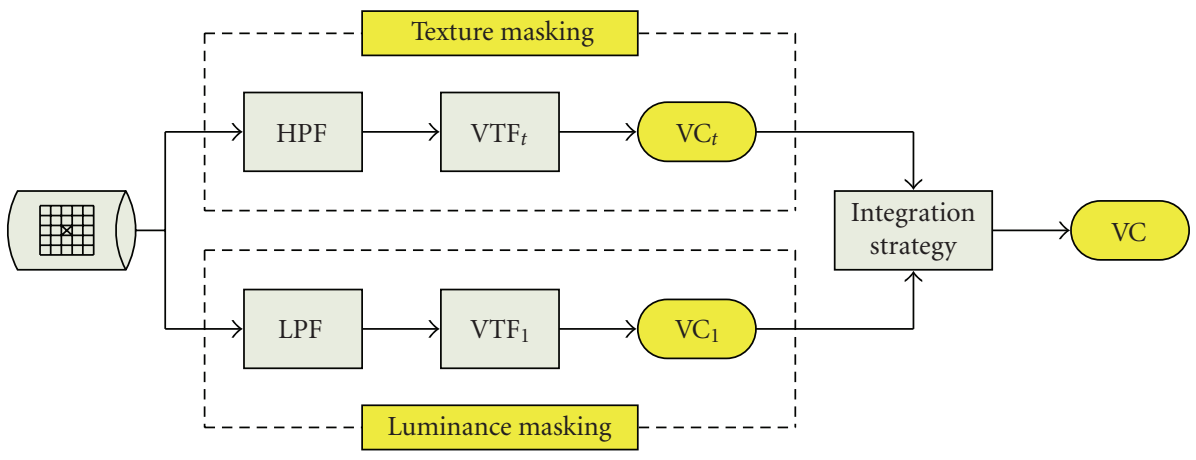

FIGURE 4: Schematic overview of the proposed human vision model.

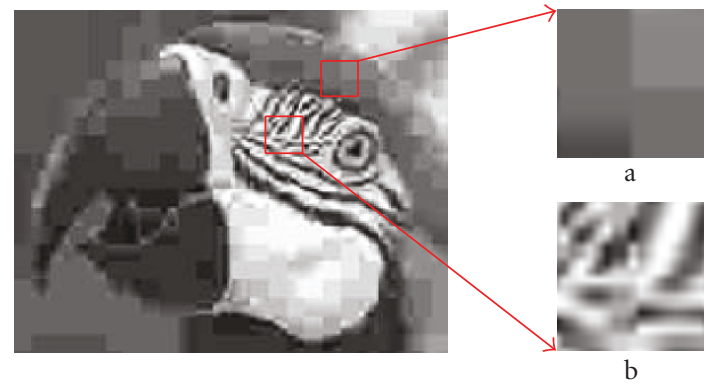

FIGURE 5: An example of texture masking on blocking artifacts.

The local luminance of a certain stimulus is calculated using a weighted low-pass filter as shown in Figure 8, in which some template coefficients are set to " 0 ." The local luminance $I_{l}(i, j)$ is given by

$$
I_{l}(i, j)=\frac{1}{26} \sum_{x=1}^{5} \sum_{y=1}^{5} I(i-3+x, j-3+y) \cdot L(x, y),
$$

where $L$ is chosen as $L 1$ for calculating the background luminance in horizontal direction and $L 2$ in vertical direction. Again, splitting up the calculation in horizontal and vertical directions, and using a modified low-pass filter, in which some template coefficients are set to 0 , is done with the application of a blockiness metric in mind.

For simplicity, the relationship between the visibility coefficient $\operatorname{VC}_{l}(i, j)$ and the local luminance $I_{l}(i, j)$ is modeled by a nonlinear function (e.g., power law) for lowbackground luminance (i.e., below 81) and is approximated by a linear function at higher background luminance (i.e., above 81). This functional behavior is shown in Figure 8 and mathematically described as

$$
\mathrm{VC}_{l}(i, j)= \begin{cases}\left(\frac{I_{l}(i, j)}{81}\right)^{1 / 2} & \text { if } 0 \leq I_{l}(i, j) \leq 81, \\ \left(\frac{1-\beta}{174}\right) \cdot\left(81-I_{l}(i, j)\right)+1 & \text { otherwise, }\end{cases}
$$

where $\operatorname{VC}_{l}(i, j)$ achieves the highest value of 1 when $I_{l}(i, j)=$ 81 , and $0<\beta<1$ ( $\beta=0.7$ in our experiments $)$ is used to adjust the slope of the linear part of this function.
2.3.3. Integration Strategy. The visibility of an artifact depends on various masking effects coexisting in the HVS. How to efficiently integrate them is an important issue in obtaining an accurate perceptual model [25]. Since masking intrinsically is a local phenomenon, the locality in the visibility of a distortion due to masking is maintained in the integration strategy of both masking effects. The resulting approach is schematically given in Figure 9. Based on the local image content surrounding a blocking artifact first the texture masking is calculated. In case the local activity in the area is larger than a given threshold (see (9)), a visibility coefficient $\mathrm{VC}_{t}$ is applied, followed by the application of a luminance masking coefficient $\mathrm{VC}_{l}$. In case the local activity in the area is low, only $\mathrm{VC}_{l}$ is applied. The application of $\mathrm{VC}_{l}$, where appropriately combined with $\mathrm{VC}_{t}$, results in an output value VC.

2.4. The Perceptual Blockiness Metric. The local pixel-based blockiness (LPB) defined in Section 2.2 is purely signal based and so does not necessarily yield perceptually consistent results. The human vision model proposed in Section 2.3 aims at removing the perceptually insignificant components due to visual masking. Integration of these two elements can be simply performed at a local level using the output of the human vision model (VC) as a weighting coefficient to scale the local pixel-based blockiness (LPB), resulting in a local perceptual blockiness metric (LPBM). Since the horizontal and vertical blocking artifacts are calculated separately, the LPBM for the block discontinuity along the horizontal direction is described as

$$
\operatorname{LPBM}_{h}(i, j)=\operatorname{VC}(i, j) \times \operatorname{LPB}_{h}(i, j),
$$

which is then averaged over all detected blocking artifacts in the entire image to determine an overall blockiness metric, that is, a no-reference perceptual blockiness metric (NPBM)

$$
\mathrm{NPBM}_{h}=\frac{1}{n} \sum_{k=1}^{n}\left[\operatorname{LPBM}_{h}(i, j)\right]_{k},
$$

where $n$ is the total number of pixels on the blocking grid of an image.

A metric $\mathrm{NPBM}_{v}$ can be similarly defined for the blockiness along the vertical direction and is simply combined 


\begin{tabular}{|r|c|c|c|c|}
\hline 1 & 2 & 0 & -2 & -1 \\
\hline 4 & 8 & 0 & -8 & -4 \\
\hline 6 & 12 & 0 & -12 & -6 \\
\hline 4 & 8 & 0 & -8 & -4 \\
\hline 1 & 2 & 0 & -2 & -1 \\
\hline \multicolumn{5}{|c}{$T 1$} \\
\end{tabular}

(a) The high-pass filters for texture detection

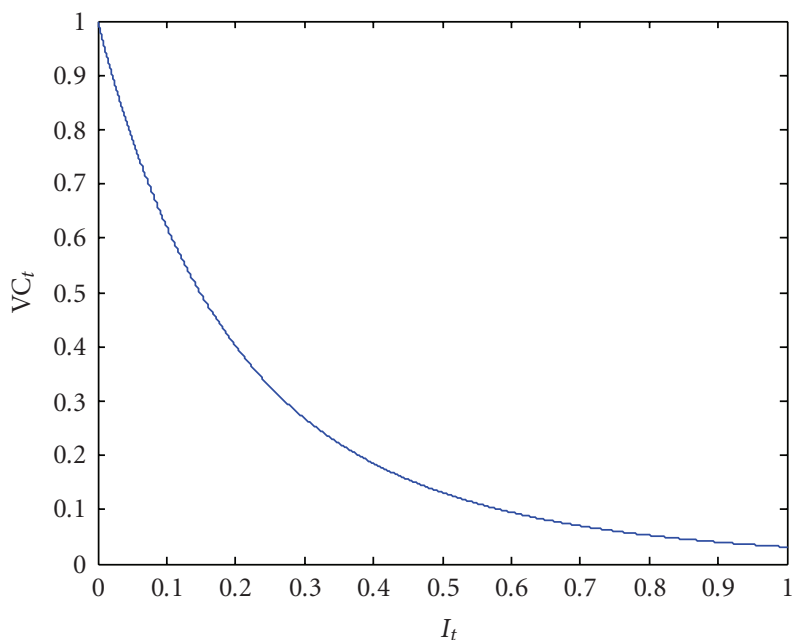

(b) Visibility transform function (VTF) used

FIGURE 6: Implementation of the texture masking.

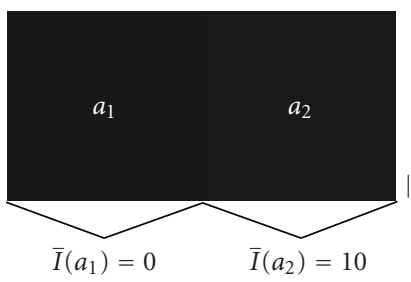

$\left|\bar{I}\left(a_{1}\right)-\bar{I}\left(a_{2}\right)\right|=10$

(a)

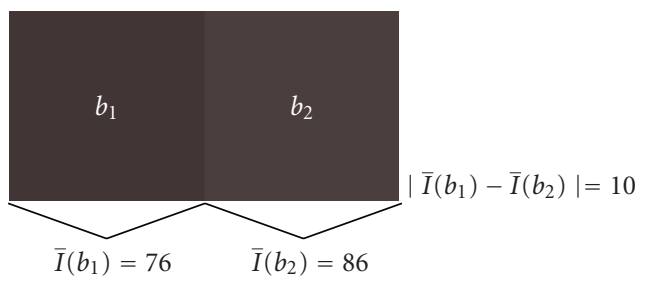

(b)

FIGURE 7: An example of luminance masking on blocking artifacts.

with $\mathrm{NPBM}_{h}$ to give the resultant blockiness score for an image. More complex combination laws may be appropriate but need to be further investigated as follows

$$
\mathrm{NPBM}=\frac{\mathrm{NPBM}_{h}+\mathrm{NPBM}_{v}}{2} .
$$

In our case, the human vision model is only calculated at the location of blocking artifact, and not for all pixels in an image. This significantly reduces the computational cost in the formulation of an overall metric.

\section{Evaluation of the Overall Metric Performance}

Subjective ratings resulting from psychovisual experiments are widely accepted as the benchmark for evaluating objective quality metrics. They reveal how well the objective

\begin{tabular}{|l|l|l|l|l|}
\hline 1 & 1 & 0 & 1 & 1 \\
\hline 1 & 2 & 0 & 2 & 1 \\
\hline 1 & 2 & 0 & 2 & 1 \\
\hline 1 & 2 & 0 & 2 & 1 \\
\hline 1 & 1 & 0 & 1 & 1 \\
\hline \multicolumn{5}{|c|}{$L 1$}
\end{tabular}

\begin{tabular}{|l|l|l|l|l|}
\hline 1 & 1 & 1 & 1 & 1 \\
\hline 1 & 2 & 2 & 2 & 1 \\
\hline 0 & 0 & 0 & 0 & 0 \\
\hline 1 & 2 & 2 & 2 & 1 \\
\hline 1 & 1 & 1 & 1 & 1 \\
\hline \multicolumn{5}{|c|}{$L 2$}
\end{tabular}

(a) The low-pass filters for local luminance detection

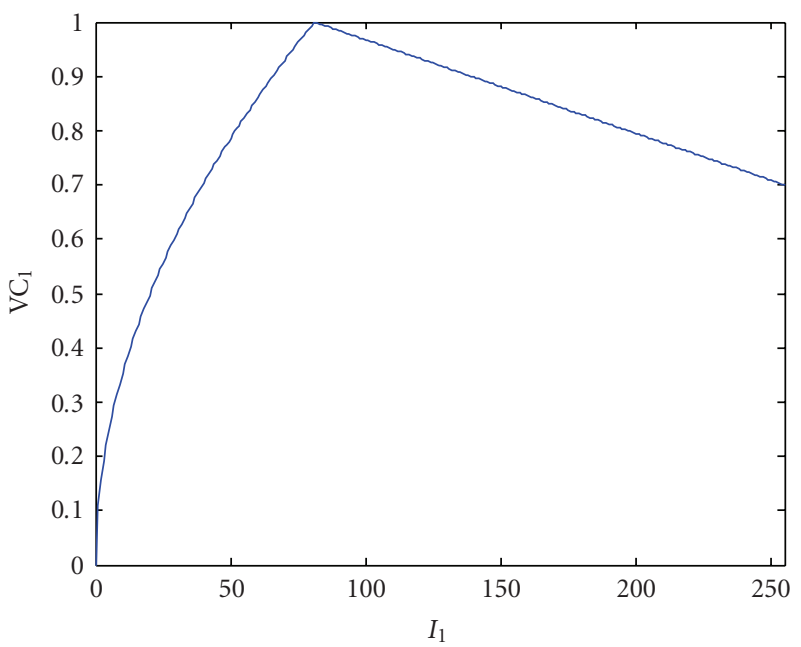

(b) Visibility transform function (VTF) used

FIGURE 8: Implementation of the luminance masking.

metrics predict the human visual experience and how to further improve the objective metrics for a more accurate mapping to the subjective data. The LIVE quality assessment database (JPEG) [26] is used to compare the performance of our proposed metric to various alternative blockiness 


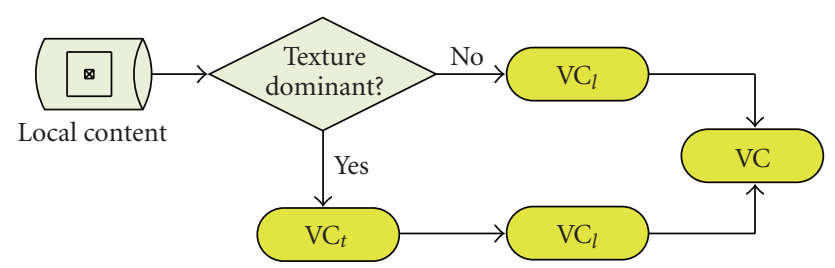

Figure 9: Integration strategy of the texture and luminance masking effect.

metrics. The LIVE database consists of a set of source images that reflect adequate diversity in image content. Twentynine high-resolution and high-quality color images are compressed using JPEG at a bit rate ranging from $0.15 \mathrm{bpp}$ to $3.34 \mathrm{bpp}$, resulting in a database of 233 images. A psychovisual experiment was conducted to assign to each image a mean opinion quality score (MOS) measured on a continuous linear scale that was divided into five intervals marked with the adjectives "Bad," "Poor,", "Fair," "Good," and "Excellent."

The performance of an objective metric can be quantitatively evaluated with respect to its ability to predict subjective quality ratings, based on prediction accuracy, prediction monotonicity, and prediction consistency [27]. Accordingly, the Pearson linear correlation coefficient, the Spearman rank order correlation coefficient, and the outlier ratio are calculated. As suggested in [27], the metric performance can also be evaluated with nonlinear correlations using a nonlinear mapping function for the objective predictions before computing the correlation. For example, a logistic function may be applied to the objective metric results to account for a possible saturation effect. This way of working usually yields higher correlation coefficients. Nonlinear correlations, however, have the disadvantage of minimizing performance differences between metrics [22]. Hence, to make a more critical comparison, only linear correlations are calculated in this paper.

The proposed overall blockiness metric, NPBM, is compared to state-of-the-art no-reference blockiness metrics based on an HVS model, namely, GBIM [15] and LABM [16]. All three metrics are applied to the LIVE database of 233 JPEG images, and their performance is characterized by the linear correlation coefficients between the subjective MOS scores and the objective metric results. Figure 10 shows the scatter plots of the MOS versus GBIM, LABM, and NPBM, respectively. The corresponding correlation results are listed in Table 1. It should be emphasized again that the correlation coefficients would be higher when allowing for a nonlinear mapping of the results of the metric to the subjective MOS. To illustrate the effect, the correlation coefficients were recalculated after applying the nonlinear mapping function recommended by VQEG [27]. In this case, GBIM, LABM, and NPBM yield the Pearson correlation coefficient of $0.928,0.933$, and 0.946 , respectively.

GBIM manifests the lowest prediction accuracy among these metrics. This is mainly due to its human vision model used, which has difficulties in handling images under demanding circumstances, for example, the highly textured

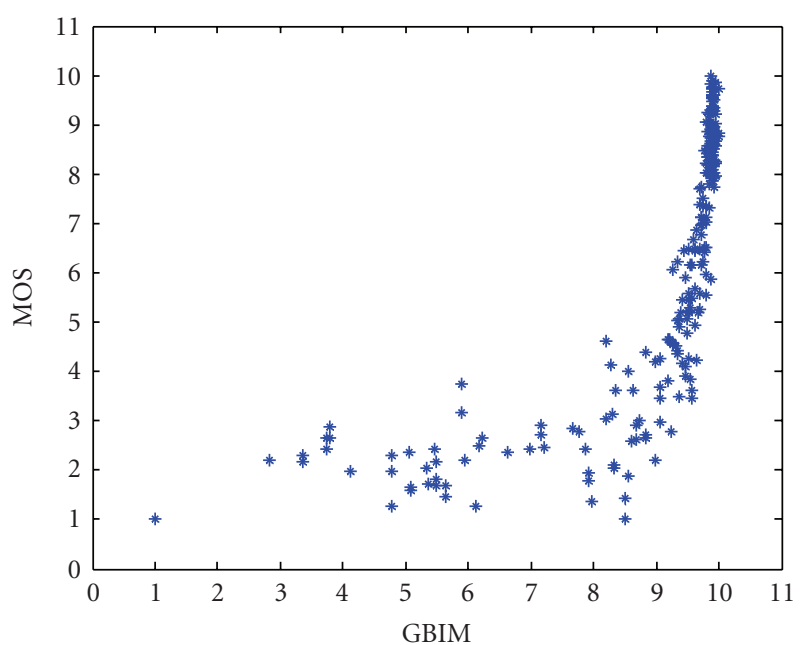

(a)

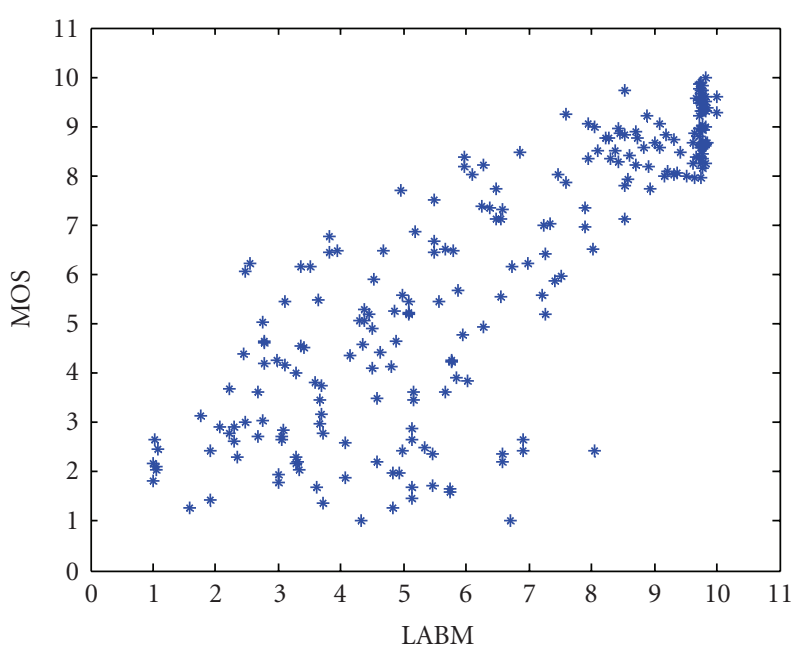

(b)

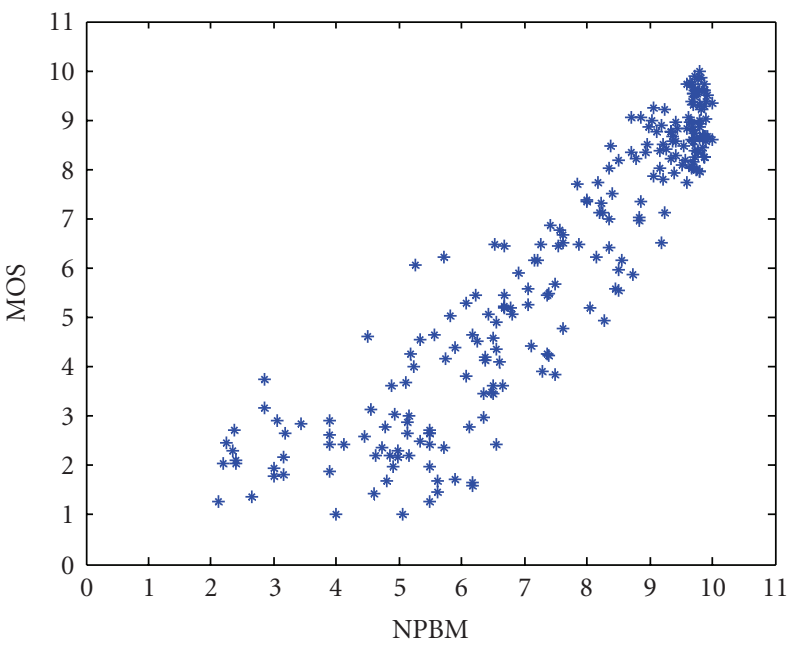

(c)

FIGURE 10: Scatter plots of MOS versus blockiness metrics. 


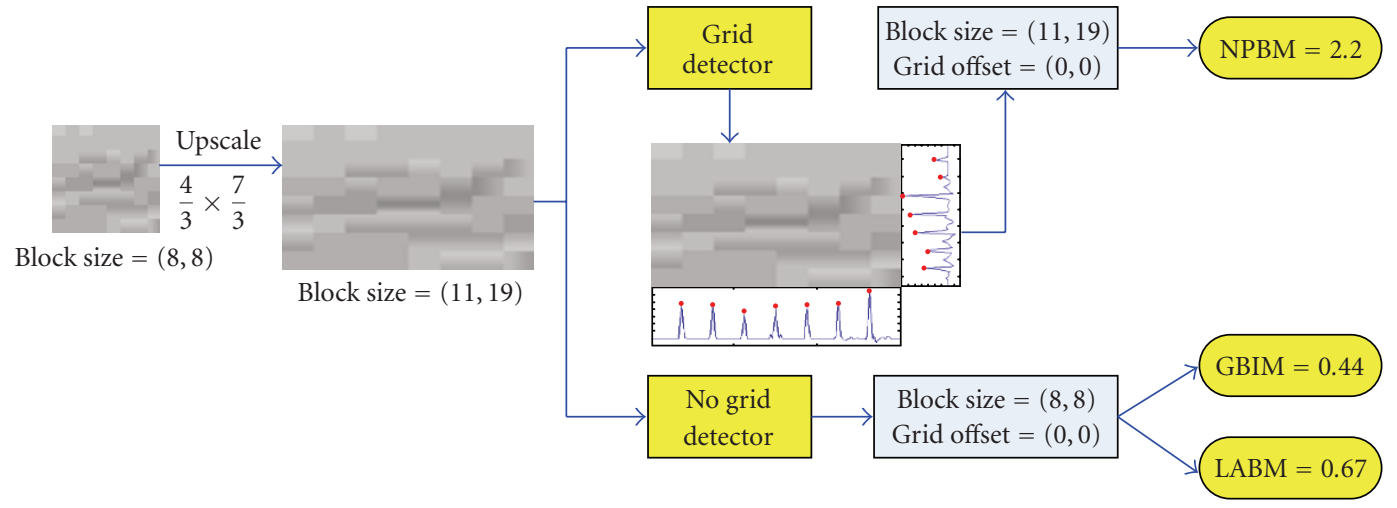

FIGURE 11: Illustration of how to evaluate the effect of a grid detector on a blockiness metric: an image patch showing visible blocking artifacts was upscaled with a scaling factor $4 / 3 \times 7 / 3$, and the metrics NPBM, GBIM, and LABM were applied to assess the blocking annoyance of the scaled image.

TABLE 1: Performance comparison of three blockiness metrics.

\begin{tabular}{lccc}
\hline Metric & Pearson linear correlation & Spearman rank-order correlation & Outlier tatio \\
\hline GBIM & 0.790 & 0.912 & 0.099 \\
LABM & 0.834 & 0.832 & 0.009 \\
NPBM & 0.918 & 0.924 & 0 \\
\hline
\end{tabular}

images in the LIVE database. LABM adopts a more flexible HVS model, that is, the JND profile with a more efficient integration of luminance and texture masking. As a consequence, the estimation of artifact visibility is more accurate for LABM than for GBIM. Additionally, LABM is based on a local estimation of blockiness, in which the distortion and its visibility due to masking are measured for each individual coding block of an image. This locally adaptive algorithm is potentially more accurate in the production of an overall blockiness score. In comparison with GBIM and LABM, our metric NPBM shows the highest prediction ability. This is primarily achieved by the combination of a refined local metric and a more efficient model of visual masking, both considering the specific structure of the artifact itself.

\section{Evaluation of Specific Metric Components}

The blocking annoyance metric, proposed in this paper, is primarily based on three aspects: (1) a grid detector to ensure the subsequent local processing; (2) a local distortion measure; (3) an HVS model for local visibility. To validate the added value of these aspects, additional experiments were conducted and a comprehensive comparison to alternatives is reported. This includes a comparison of

(i) metrics with and without a grid detector;

(ii) the local versus global approach;

(iii) metrics with and without an HVS model;

(iv) different HVS models.

4.1. Metrics with and without a Grid Detector. Our metric includes a grid detection algorithm to determine the exact location of the blocking artifacts, and thus to ensure the calculation of the metric at the appropriate pixel positions. It avoids the risk of estimating blockiness at wrong pixel positions, for example, in scaled images. To illustrate the problem of blockiness estimation in scaled images, a small experiment was conducted. As illustrated in Figure 11, an image patch of $64 \times 64$ pixels was extracted from a low bit-rate $(0.34 \mathrm{bpp})$ JPEG image of the LIVE database. This image patch had a grid of blocks of $8 \times 8$ pixels starting at its top-left corner, and it clearly exhibited visible blocking artifacts. It was scaled up with a factor $4 / 3 \times 7 / 3$, resulting in an image with an effective block size of $11 \times 19$ pixels. Blocking annoyance in this scaled image was estimated with three metrics, that is, NPBM, GBIM, and LABM. Due to the presence of a grid detector, the NPBM yielded a reasonable score of 2.2 (NPBM scores range from 0 (no blockiness) to 10 for the highest blocking annoyance). However, in the absence of a grid detector, both GBIM and LABM did not detect any substantial blockiness; they had a score of GBIM $=0.44$ and $\mathrm{LABM}=0.67$, which corresponds to "no blockiness" according to their scoring scale (see, $[15,16]$ ). Thus, GBIM and LABM fail in predicting blocking annoyance of scaled images, mainly due to the absence of a grid detector. Clearly, these metrics could benefit in a similar way as our own metric from including the location of the grid.

Various alternative grid detectors are available in literature. They all rely on the gradient image to detect the blocking grid. To do so, they either calculate the FFT for each single row and column of an image [13] or they calculate the normalized gradient for every pixel in its two dimensions [9]. Especially, for large images (e.g., in the case of HD-TV), these operations are computationally expensive. The main advantage of our proposed grid detector lies in its simplicity, 


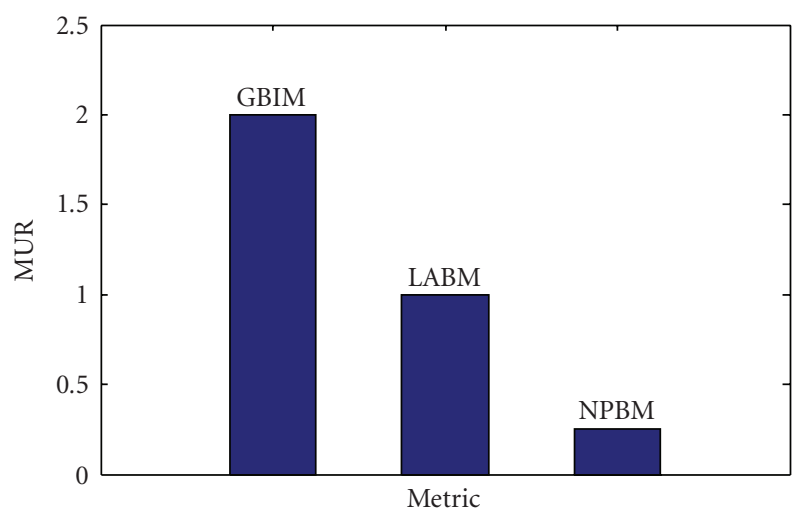

FIGURE 12: Comparison of the computational cost of three metrics, using model utilization ratio (MUR).

compared to existing alternatives in literature. Such as in the approach reported in [18], we first project the gradient image into a 1-D signal and then enhance the signal maxima using once a median filter. In addition, the size and offset of the grid are extracted from the resulting 1-D signal using a DFT. The latter is less computationally expensive than the approach chosen in [18], being a complex maximumlikelihood method.

Apart from affecting the blocking grid position, scaling may also affect the blocking artifact visibility [9]. This aspect, however, is not yet taken into account in our proposed metric.

4.2. Local versus Global Approach. The difference in local versus global approach can be best understood by comparing their basic formulation. A local metric, as proposed in this paper, is based on a general formulation of the form MF1:

$$
\mathrm{MF} 1=\frac{1}{n} \sum_{k=1}^{n}[\mathrm{LPB}(k) \times M(k)]
$$

where $k$ denotes the pixel location of blocking artifacts, and LPB and $M$ denote the local pixel-based blockiness (see (7)) and the HVS model embedded, respectively. Both of them are calculated locally within a region of the image centered on individual blocking artifacts.

A global metric as, for example, used in GBIM [15] is based on a general formulation of the form MF2:

$$
\mathrm{MF} 2=\frac{\left\|G(i, j)_{\text {block-edge }} \times M(i, j)_{\text {block-edge }}\right\|}{\left\|G(i, j)_{\text {non-block-edge }} \times M(i, j)_{\text {non-block-edge }}\right\|},
$$

where $G$ denotes the interpixel difference (see (1)), $M$ denotes the HVS model embedded, and $\|\cdot\|$ is the L2norm. The numerator is calculated at the location of blocking artifacts, while the denominator is calculated for pixels which are not on the blocking grid.

An obvious advantage of the local approach over the global approach is already revealed by their formulation: MF1 only calculates the HVS model for pixels on the blocking grid, while MF2 needs to calculate the HVS model for all pixels in the image. Since the major cost of an HVSbased blockiness metric is usually introduced by the human vision model, reducing the number of times the HVS model calculated in the whole process is highly beneficial for the computational load. The computational cost related to the number of times the HVS model has to be calculated in a metric can be quantified by means of a model utilization ratio (MUR), which is simply defined as the total number of times $T_{M}$ that the HVS model is computed, divided over the total number of pixels $M \times N$ in the image

$$
\text { MUR }=\frac{T_{M}}{M \times N}
$$

Evidently, the lower this ratio, the simpler the metric is.

Figure 12 shows the MUR for GBIM, LABM, and NPBM, respectively. Both GBIM and LABM calculate the human vision model for every pixel in an image, which yields a MUR of 1. For GBIM the MUR is increased by a factor of 2 , since masking is estimated for the horizontal and vertical blockiness directions separately. For our metric the MUR is only 0.25 in case of a block size of $8 \times 8$ pixels, which is a direct result of calculating the HVS model only at detected blocking artifacts. This implies that when neglecting the difference in computational cost between the various HVS models for a moment, the computational load of NPBM is reduced by approximately $7 / 8$ with respect to GBIM and by $3 / 4$ with respect to $\mathrm{LABM}$.

Of course, in this respect also the complexity of the HVS model used needs to be taken into account. This is further discussed in Section 4.4, taking into account various HVS models. Additionally, there also is a performance difference between the local and global approaches. But, since the performance gain depends on the specific choice of HVS used, this point is also discussed in Section 4.4.

4.3. Metrics with and without an HVS Model. To validate the added value of including an HVS model in a blockiness metric, we compared our proposed HVS-based metric NPBM to the state-of-the-art non-HVS-based metric of [9], which is referred to as NBAM. NBAM is also a global metric formulated according to (18), but instead of using an HVS model, it replaces the interpixel difference by the relative gradient in order to determine the visual strength of a block discontinuity. It was achieved a promising performance over the entire LIVE database as indicated by the Pearson correlation coefficient (after nonlinear regression) of 0.92 , which is comparable to our metric with a Pearson correlation coefficient of 0.94 . However, because of the absence of an HVS model, the robustness of NBAM against image content might be an issue. It may be doubted to what extent the objective metric is able to predict blockiness in more demanding images, for example, for a set of highly textured images, compressed at very low bit-rates, for which visual masking is important.

To evaluate this, a subset of six highlytextured images, as shown in Figure 13, was selected from the twentynine source images of the LIVE database. Including different compression levels, this resulted in a test database of 50 


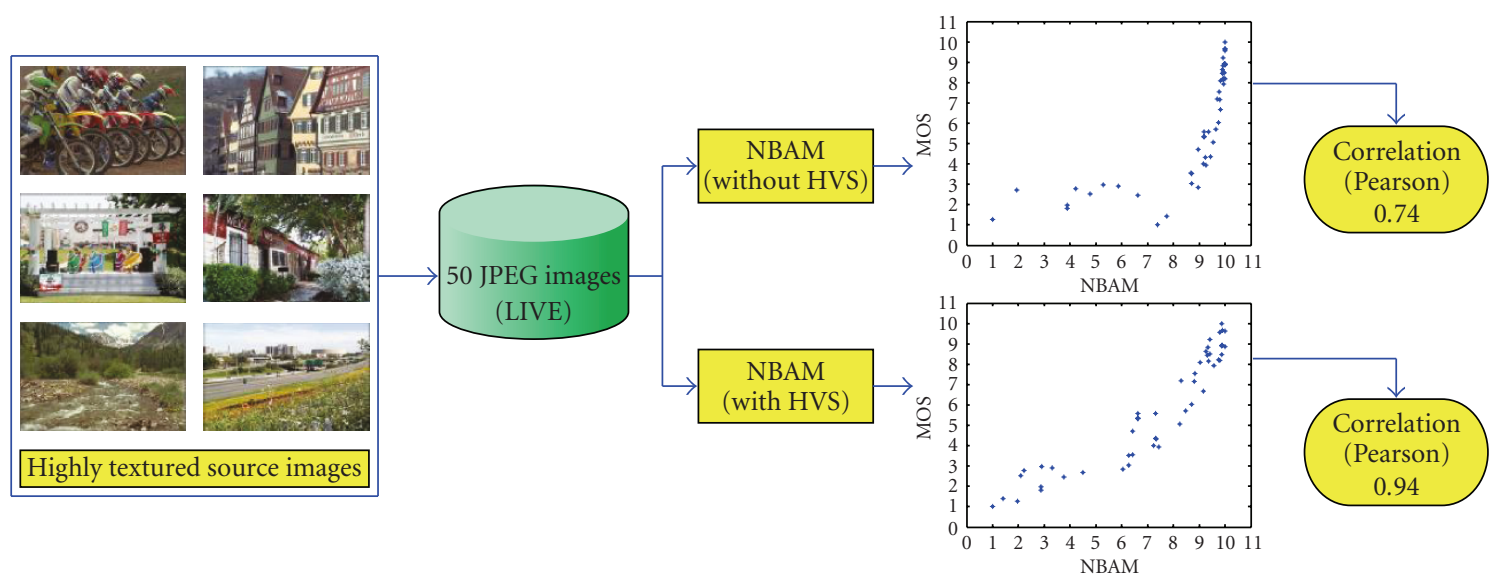

FIGURE 13: Illustration of the added value of including an HVS model in a blockiness metric: a database of 50 highly textured JPEG images was extracted from the LIVE database, and blockiness annoyance was estimated with the metrics NBAM (without HVS) and NPBM (with HVS). The prediction performance is given in terms of the Pearson correlation coefficient.

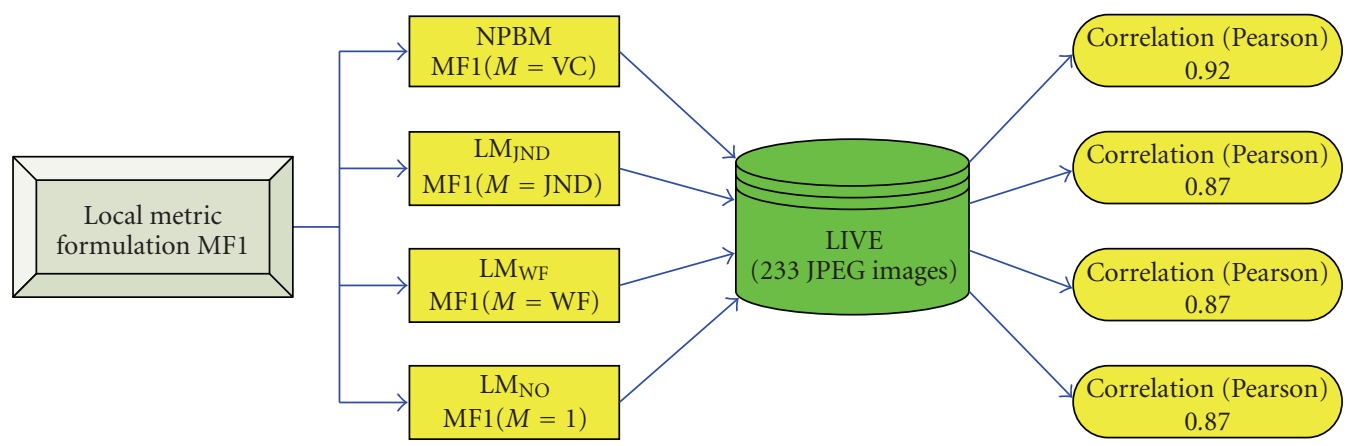

FIGURE 14: Illustration of the comparison of various HVS models: a blockiness metric (i.e., MF1) having four optional HVS models embedded is tested with the LIVE database, and the performance for each resulting metric is quantified by the Pearson correlation coefficient.

JPEG images with their corresponding MOS scores extracted from the LIVE database. For these images, texture masking was dominant, that is, most blocking artifacts were largely masked by background nonuniformity.

The blockiness metrics, NPBM and NBAM, were applied to this test database. Their prediction performance is quantified by the Pearson correlation coefficient (without nonlinear regression) as illustrated in Figure 13. As expected, the simple metric NBAM fails in accurately predicting the subjective ratings of this subset of demanding images, mainly due to the lack of an HVS model. NPBM shows a robust prediction ability, resulting in a high correlation with the subjective MOS.

4.4. Comparison of Different HVS Models. To compare the added value of our proposed HVS model to existing alternatives, various HVS models $M$ have been embedded in the general formulation of our local metric (see MF1 (17)). For $M$ we used four alternatives:

(i) VC model (i.e., our proposed HVS model);

(ii) JND model (i.e., the JND profile model based on $[21])$; (iii) WF model (i.e., the HVS model used in GBIM [15]);

(iv) $M=1$ model (i.e., no HVS model embedded).

Doing so, resulted in four blockiness metrics, which we refer to as $\mathrm{LM}_{\mathrm{VC}}$ (i.e., NPBM), $\mathrm{LM}_{\mathrm{JND}}, \mathrm{LM}_{\mathrm{WF}}$, and $\mathrm{LM}_{\mathrm{NO}}$, respectively. These four metrics were applied to the LIVE database of 233 JPEG images. The metric performance was quantified by the Pearson correlation coefficient (without nonlinear regression) as illustrated in Figure 14. In such a scenario, the performance difference between any two metrics can be attributed to the HVS model embedded. $\mathrm{LM}_{\mathrm{NO}}$ (i.e., MF1 without any HVS model) is used as the benchmark, and the HVS model gain is determined by calculating the difference in Pearson correlation coefficient between the metric $\mathrm{LM}_{\mathrm{NO}}$ and any of the other three metrics.

Figure 14 clearly illustrates that our HVS model yields the biggest gain compared to the other three alternatives. For the local approach defined as MF1 in (17), there is no added value of using the JND or WF model in the metric, since their performance is comparable to that of the metric without HVS model. This may, of course, be due to the fact that the JND and WF models were not designed to be combined with our proposed local metric. Our VC model, on the other hand, is designed together with the definition of MF1, and as 


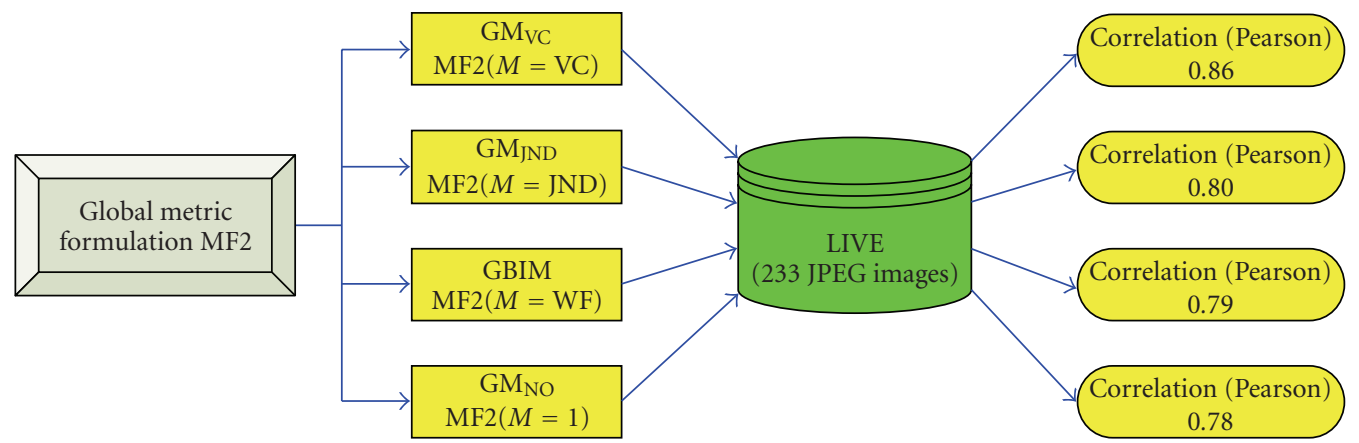

FIgURE 15: Illustration of the comparison of various HVS models: a blockiness metric (i.e., MF2) having four optional HVS models embedded is tested with the LIVE database, and the performance for each resulting metric is quantified by the Pearson correlation coefficient.

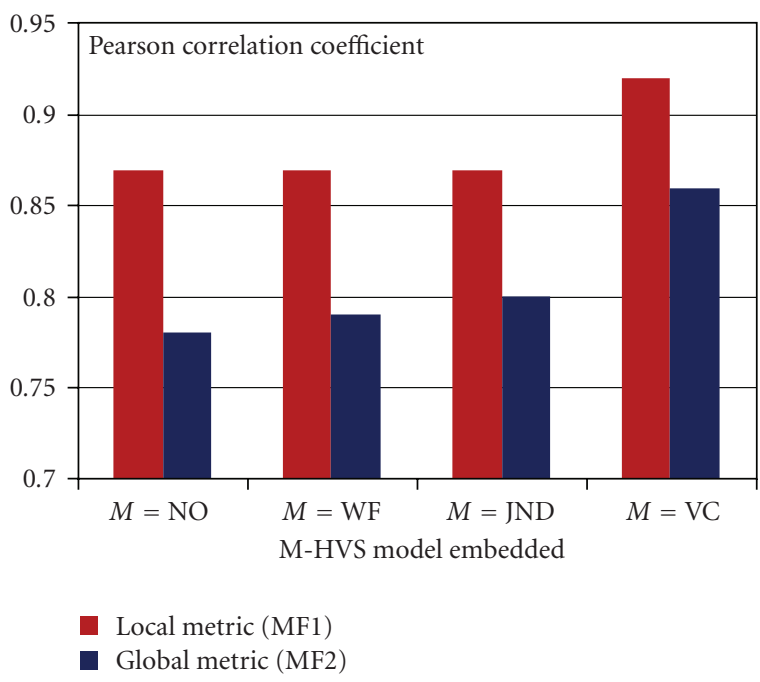

Figure 16: Comparison of the local and global approaches to a blockiness metric, and of metrics with different HVS models embedded.

a result a high correlation coefficient is found for the NPBM metric.

To investigate whether our HVS model is also valuable for traditionally used global metrics (see MF2 in (18)), the same experiment was repeated by substituting in MF2 the four options for $M$. This yielded another set of four blockiness metrics, which are referred to as $\mathrm{GM}_{\mathrm{VC}}, \mathrm{GM}_{\mathrm{JND}}$, $\mathrm{GM}_{\mathrm{WF}}$ (i.e., GBIM), and $\mathrm{GM}_{\mathrm{NO}}$, respectively. Their performance when applied to the LIVE database is illustrated in Figure 15.

It illustrates that also for a global metric our HVS model has the largest added value. In this case, however, also the WF and JND models have some added value. It should be noted, however, that in our evaluations the WF and JND models were implemented as described in the original publications (i.e., $[15,21])$. Some parameters in the implementations may be adjusted specifically to the LIVE database to provide a better correlation.

To summarize, the contribution of our proposed HVS model to a blockiness metric is consistently shown, independent of the specific design of the blockiness metric. In addition, a number of significant simplifications used in our HVS model are already discussed in Section 2.3. The complexity of our VC model is comparable to that of the WF model, both of them use a simple weighting function for local visibility. However, the JND model is a rather complex HVS model, mainly due to the difficulties in estimating the visibility thresholds for various masking effects and in combing different JND thresholds. The simplicity of the VC model itself, coupled with its specific design for a local approach to avoid calculating it on irrelevant pixels, consequently makes this HVS model especially promising in terms of real-time applications.

An additional interesting finding from the comparison of Figures 14 and 15 is that there is indeed a gain in performance applying the MF1 formulation (local approach) instead of the MF2 formulation (global approach), independent of the HVS model used. In the absence of any HVS model, the gain of MF1 over MF2 (i.e., from $\mathrm{LM}_{\mathrm{NO}}$ to $\mathrm{GM}_{\mathrm{NO}}$ ) corresponds to an increase in the Pearson correlation coefficient from 0.78 to 0.87. For the other HVS models, the corresponding numbers are summarized in Figure 16. It confirms that a promising performance is achieved when applying the local approach in a blockiness metric.

\section{Conclusions}

In this paper, a novel blockiness metric to assess blocking annoyance in block-based DCT coding is proposed. It is based on the following features.

(i) A simple grid detector to ensure the effectiveness of the blockiness metric and to account for deviations in the blocking grid of the incoming signal or as a consequence of spatial scaling.

(ii) A local pixel-based blockiness value that measures the strength of the distortion within a region of the image centered around each individual blocking artifact.

(iii) A simplified and more efficient model of visual masking, exhibiting an improved robustness in terms of content independency, and allowing suprathreshold estimation of perceived annoyance. 
An advantage of the proposed approach, especially in case of real-time application, is that the additional computational cost introduced by the HVS is largely reduced by eliminating calculations of the human vision model for nonrelevant pixels. This is primarily accomplished taking advantage of the locality of both the pixel-based blockiness value and the visibility model. Nonetheless, the metric is mainly used to assess overall blockiness annoyance, which is simply done by summing the local contributions over the whole image.

Experimental results show that our proposed blockiness metric results in a strong correlation with subjective data and outperforms state-of-the-art metrics in terms of prediction accuracy. Combined with its practical reliability and computational efficiency, our metric is a good alternative for realtime implementation.

\section{References}

[1] Z. Wang and A. C. Bovik, Modern Image Quality Assessment, Synthesis Lectures on Image, Video, \& Multimedia Processing, Morgan \& Claypool, San Rafael, Calif, USA, 2006.

[2] C. C. Koh, S. K. Mitra, J. M. Foley, and I. E. J. Heynderickx, "Annoyance of individual artifacts in MPEG-2 compressed video and their relation to overall annoyance," in Human Vision and Electronic Imaging X, vol. 5666 of Proceedings of SPIE, pp. 595-606, San Jose, Calif, USA, January 2005.

[3] S. Winkler, "Issues in vision modeling for perceptual video quality assessment," Signal Processing, vol. 78, no. 2, pp. 231252, 1999.

[4] Z. Yu and H. R. Wu, "Human visual system based objective digital video quality metrics," in Proceedings of the 5th International Conference on Signal Processing (WCCC-ICSP '00), vol. 2, pp. 1088-1095, Beijing, China, August 2000.

[5] Z. Yu, H. R. Wu, S. Winkler, and T. Chen, "Vision-modelbased impairment metric to evaluate blocking artifacts in digital video," Proceedings of the IEEE, vol. 90, no. 1, pp. 154169, 2002.

[6] E. M. Yeh, A. C. Kokaram, and N. G. Kingsburg, "Perceptual distortion measure for edgelike artifacts in image sequences," in Human Vision and Electronic Imaging III, vol. 3299 of Proceedings of SPIE, pp. 160-172, San Jose, CA, USA, January 1998.

[7] S. A. Karunasekera and N. G. Kingsbury, "Distortion measure for blocking artifacts in images based on human visual sensitivity," IEEE Transactions on Image Processing, vol. 4, no. 6, pp. 713-724, 1995.

[8] M. Yuen and H. R. Wu, "A survey of hybrid MC/DPCM/DCT video coding distortions," Signal Processing, vol. 70, no. 3, pp. 247-278, 1998.

[9] R. Muijs and I. Kirenko, "A no-reference blocking artifact measure for adaptive video processing," in Proceedings of the 13th European Signal Processing Conference (EUSIPCO '05), Antalya, Turkey, September 2005.

[10] I. O. Kirenko, R. Muijs, and L. Shao, "Coding artifact reduction using non-reference block grid visibility measure," in Proceedings of the IEEE International Conference on Multimedia and Expo (ICME '06), pp. 469-472, Toronto, Canada, July 2006.

[11] Z. Wang, H. R. Sheikh, and A. C. Bovik, "No reference perceptual quality assessment of JPEG compressed images," in Proceedings of the IEEE International Conference on Image
Processing (ICIP '02), vol. 1, pp. 477-480, Rochester, NY, USA, September 2002.

[12] R. V. Babu, S. Suresh, and A. Perkis, "No-reference JPEGimage quality assessment using GAP-RBF," Signal Processing, vol. 87, no. 6, pp. 1493-1503, 2007.

[13] Z. Wang, A. C. Bovik, and B. L. Evans, "Blind measurement of blocking artifacts in images," in Proceedings of the IEEE International Conference on Image Processing (ICIP '00), vol. 3, pp. 981-984, Vancouver, Canada, September 2000.

[14] S. Liu and A. C. Bovik, "Efficient DCT-domain blind measurement and reduction of blocking artifacts," IEEE Transactions on Circuits and Systems for Video Technology, vol. 12, no. 12, pp. 1139-1149, 2002.

[15] H. R. Wu and M. Yuen, "A generalized block-edge impairment metric for video coding," IEEE Signal Processing Letters, vol. 4, no. 11, pp. 317-320, 1997.

[16] F. Pan, X. Lin, S. Rahardja, et al., "A locally adaptive algorithm for measuring blocking artifacts in images and videos," Signal Processing: Image Communication, vol. 19, no. 6, pp. 499-506, 2004.

[17] E. Lesellier and J. Jung, "Robust wavelet-based arbitrary grid detection for MPEG," in Proceedings of the IEEE International Conference on Image Processing (ICIP'02), vol. 3, pp. 417-420, Rochester, NY, USA, September 2002.

[18] S. Tjoa, W. S. Lin, H. V. Zhao, and K. J. R. Liu, "Block size forensic analysis in digital images," in Proceedings of the IEEE International Conference on Acoustics, Speech, and Signal Processing (ICASSP '07), vol. 1, pp. 633-636, Honolulu, Hawaii, USA, April 2007.

[19] H. Liu and I. Heynderickx, "A no-reference perceptual blockiness metric," in Proceedings of the IEEE International Conference on Acoustics, Speech, and Signal Processing (ICASSP '08), pp. 865-868, Las Vegas, Nev, USA, March-April 2008.

[20] T. N. Pappas and R. J. Safranek, "Perceptual criteria for image quality evaluation," in Handbook of Image and Video Processing, pp. 669-684, Academic Press, New York, NY, USA, 2000.

[21] C.-H. Chou and Y.-C. Li, "A perceptually tuned subband image coder based on the measure of just-noticeabledistortion profile," IEEE Transactions on Circuits and Systems for Video Technology, vol. 5, no. 6, pp. 467-476, 1995.

[22] S. Winkler, Vision models and quality metrics for image processing applications, Ph.D. dissertation, Department of Electrical Engineering, EPFL, Lausanne, Switzerland, 2002.

[23] K. I. Laws, "Texture energy measures," in Proceedings of the DARPA Image Understanding Workshop, pp. 47-51, Los Angeles, Calif, USA, November 1979.

[24] B. Girod, "The information theoretical significance of spatial and temporal masking in video signals," in Human Vision, Visual Processing, and Digital Display, vol. 1077 of Proceedings of SPIE, pp. 178-187, Los Angeles, Calif, USA, January 1989.

[25] X. Yang, W. Lin, Z. Lu, E. Ong, and S. Yao, "Motioncompensated residue preprocessing in video coding based on just-noticeable-distortion profile," IEEE Transactions on Circuits and Systems for Video Technology, vol. 15, no. 6, pp. 742-751, 2005.

[26] H. R. Sheikh, Z. Wang, L. Cormack, and A. C. Bovik, "LIVE image quality assessment database Release 2," March 2008, http://live.ece.utexas.edu/research/quality.

[27] VQEG, "Final report from the video quality experts group on the validation of objective models of video quality assessment," Tech. Rep., Video Quality Experts Group, Ottawa, Canada, August 2003, http://www.its.bldrdoc.gov/vqeg. 